\title{
Observability of spherical photon orbits in near-extremal Kerr black holes
}

\author{
Takahisa Igata, Hideki Ishihara, and Yu Yasunishi
}

\begin{tabular}{|c|l|}
\hline Citation & Physical Review D, 100(4);044058 \\
\hline Issue Date & 2019-08-15 \\
\hline Type & Journal Article \\
\hline Textversion & Publisher \\
\hline Rights & $\begin{array}{l}\text { CAmerican Physical Society. This article may be downloaded for personal use only. Any } \\
\text { other use requires prior permission of the author and American Physical Society. } \\
\text { The following article appeared in Phys. Rev. D 100, 044058 and may be found at } \\
\text { https://doi.org/10.1103/PhysRevD.100.044058. }\end{array}$ \\
\hline DOI & 10.1103/PhysRevD.100.044058 \\
\hline
\end{tabular}

Self-Archiving by Author(s)

Placed on: Osaka City University 


\title{
Observability of spherical photon orbits in near-extremal Kerr black holes
}

\author{
Takahisa Igata, ${ }^{1, *}$ Hideki Ishihara, ${ }^{2, \dagger}$ and Yu Yasunishi ${ }^{2}$ \\ ${ }^{1}$ Department of Physics, Rikkyo University, Toshima, Tokyo 171-8501, Japan \\ ${ }^{2}$ Department of Mathematics and Physics, Graduate School of Science, Osaka City University, \\ Osaka, 558-8585, Japan
}

(Received 5 April 2019; published 29 August 2019)

\begin{abstract}
We investigate the spherical photon orbits in near-extremal Kerr spacetimes. We show that the spherical photon orbits with impact parameters in a finite range converge on the event horizon. Furthermore, we demonstrate that the Weyl curvature near the horizon does not generate the shear of a congruence of such light rays. Because of this property, a series of images produced by the light orbiting around a near-extremal Kerr black hole several times can be observable.
\end{abstract}

DOI: 10.1103/PhysRevD.100.044058

\section{INTRODUCTION}

A large number of gravitational lens systems have been observed in our Universe [1]. In the case of the gravitational lensing by a black hole, it would be possible that we observe a series of images produced by a direct light, light orbiting around the black hole once, twice, and so on. The series of images are related to the unstable photon orbits with constant radii around the black hole, the so-called spherical photon orbits [2]. The photons escaping from the spherical photon orbits toward an observer make the series of images.

Very recently, the Event Horizon Telescope Collaboration reported observations of a bright emission ring in the central region of M87 [3-8] by using a global very long baseline interferometry array. It is interpreted that the observed ring is produced by photons orbiting the supermassive black hole sitting in the center of M87.

There are a lot of black hole candidates, and many of them are thought to be rapidly rotating black holes [9]. Therefore, it is important to investigate the gravitational lensing by rapidly rotating black holes. It is well known that the radius of the prograde circular photon orbit on the equatorial plane of a Kerr black hole approaches the horizon radius in the extremely rotating limit. If we can observe the images related to such photon orbits, we obtain information on the near-horizon geometry of the extremal Kerr black hole.

In a slowly rotating black hole case, it would be hard to observe the images produced by light orbiting around the black hole because the brightness of the images decreases as the light ray winds. The Weyl curvature around the black hole generates the shear of a congruence of the light rays,

\footnotetext{
*igata@rikkyo.ac.jp

ishihara@sci.osaka-cu.ac.jp
}

and the shear induces the expansion of the congruence. Hence, the brightness of the images decreases exponentially as the number of windings increases.

In an extremely rotating black hole case, it is known that the near-horizon geometry with long throat structure admits enhanced symmetry $[10,11]$. Recently, in the context of the Kerr/CFT correspondence [12,13], the near-horizon region of the near-extremal Kerr black hole has been an interesting area that provides new phenomena. Then, we suppose that spherical photon orbits that exist in the near-horizon region in the near-extremal Kerr black hole have different properties from the ones in a slowly rotating black hole [14,15]. We show, in this article, that the Weyl curvature does not generate the shear of a congruence of the spherical photon orbits near the horizon of near-extremal Kerr black holes.

\section{SPHERICAL PHOTON ORBITS IN Kerr SPACETIMES}

The Kerr metric in the Boyer-Lindquist coordinates $(t, r, \theta, \varphi)$ is given by

$$
\begin{aligned}
g_{\mu \nu} \mathrm{d} x^{\mu} \mathrm{d} x^{\nu}= & -\frac{\Delta \Sigma}{A} \mathrm{~d} t^{2}+\frac{\Sigma}{\Delta} \mathrm{d} r^{2}+\Sigma \mathrm{d} \theta^{2} \\
& +\frac{A}{\Sigma} \sin ^{2} \theta\left[\mathrm{d} \varphi-\frac{2 M a r}{A} \mathrm{~d} t\right]^{2}, \\
\Sigma= & r^{2}+a^{2} \cos ^{2} \theta, \\
\Delta= & r^{2}+a^{2}-2 M r, \\
A= & \left(r^{2}+a^{2}\right)^{2}-a^{2} \Delta \sin ^{2} \theta .
\end{aligned}
$$

When $|a| \leq M$, the metric describes a rotating black hole with mass $M$ and specific angular momentum $a$. The black hole spacetime has the event horizon at 
$r=r_{+}:=M+\sqrt{M^{2}-a^{2}}$ and the inner horizon at $r=r_{-}:=M-\sqrt{M^{2}-a^{2}}$. The event horizon is generated by the null tangent vector field

$$
\chi^{a}=(\partial / \partial t)^{a}+\Omega_{\mathrm{h}}(\partial / \partial \varphi)^{a},
$$

where $\Omega_{\mathrm{h}}=a /\left(r_{+}^{2}+a^{2}\right)$ is the angular velocity of the event horizon. We use units in which $M=1$ in what follows.

Let $k^{a}$ be a tangent vector to the null geodesics parametrized by an affine parameter $\lambda$ in the Kerr spacetime. According to the time translation symmetry and axisymmetry, $E=-k_{t}$ and $L=k_{\varphi}$ are constants of motion. In addition, we have a constant of motion [16],

$$
Q=K_{a b} k^{a} k^{b}-(L-a E)^{2},
$$

where $K_{a b}$ is the Killing tensor defined by

$$
\begin{aligned}
K_{a b}= & \Sigma^{2}(\mathrm{~d} \theta)_{a}(\mathrm{~d} \theta)_{b}+\sin ^{2} \theta\left[\left(r^{2}+a^{2}\right)(\mathrm{d} \varphi)_{a}-a(\mathrm{~d} t)_{a}\right] \\
& \times\left[\left(r^{2}+a^{2}\right)(\mathrm{d} \varphi)_{b}-a(\mathrm{~d} t)_{b}\right]-a^{2} \cos ^{2} \theta g_{a b} .
\end{aligned}
$$

Introducing the dimensionless impact parameters

$$
b=\frac{L}{E}, \quad q=\frac{Q}{E^{2}},
$$

for nonvanishing $E$, since $K_{a b} k^{a} k^{b} \geq 0$, we have the inequality

$$
q+(b-a)^{2} \geq 0
$$

In terms of the parameters $b$ and $q$, the null geodesic equations are

$$
k^{t}=\dot{t}=\frac{1}{\Sigma}\left[a\left(b-a \sin ^{2} \theta\right)+\frac{r^{2}+a^{2}}{\Delta}\left[r^{2}+a(a-b)\right],\right.
$$$$
k^{r}=\dot{r}=\frac{\sigma_{r}}{\Sigma} \sqrt{-V},
$$

$k^{\theta}=\dot{\theta}=\frac{\sigma_{\theta}}{\Sigma} \sqrt{-U}$,

$k^{\phi}=\dot{\varphi}=\frac{1}{\Sigma}\left[\frac{b}{\sin ^{2} \theta}-a+\frac{a}{\Delta}\left[r^{2}+a(a-b)\right]\right]$,

where $\sigma_{r}, \sigma_{\theta}= \pm 1$, the dots denote derivatives with respect to $\lambda$, and functions $V, U$ are given by

$$
\begin{gathered}
V=\Delta\left[q+(b-a)^{2}\right]-\left[r^{2}+a(a-b)\right]^{2}, \\
U=\cos ^{2} \theta\left(\frac{b^{2}}{\sin ^{2} \theta}-a^{2}\right)-q .
\end{gathered}
$$

From the equation of motion for $\theta$ in Eq. (8), $U \leq 0$ should hold, and then, the allowed region of $\theta$ is classified into the following three cases:

(i) If $q>0$, then $|\cos \theta| \leq u_{+}$,

(ii) If $q=0$ and $b^{2}>a^{2}$, then $\theta=\pi / 2$,

(iii) If $q \leq 0$ and $b^{2} \leq(a-\sqrt{-q})^{2}$,

$$
\text { then } u_{-} \leq|\cos \theta| \leq u_{+} \text {, }
$$

where

$u_{ \pm}=\frac{1}{\sqrt{2} a}\left[a^{2}-b^{2}-q \pm \sqrt{\left(a^{2}-b^{2}-q\right)^{2}+4 a^{2} q}\right]^{1 / 2}$.

In a special case $(b, q)=\left(a \sin ^{2} \theta_{0},-a^{2} \cos ^{4} \theta_{0}\right)$ in (iii), where $\theta_{0}$ is a constant, $k^{a}$ is identified with the principal null vectors in the Kerr spacetime:

$$
N_{ \pm}^{a}=\frac{r^{2}+a^{2}}{\Delta}(\partial / \partial t)^{a} \pm(\partial / \partial r)^{a}+\frac{a}{\Delta}(\partial / \partial \varphi)^{a} .
$$

Hereafter, we focus on the spherical photon orbits [2], $\dot{r}=0$ and $\ddot{r}=0$, in the range $r_{+}<r$ or $0<r<r_{-}$. Then, the radial equation in Eq. (8) leads to the equations

$$
V=0, \quad \frac{\mathrm{d} V}{\mathrm{~d} r}=0 .
$$

Solving coupled algebraic equations (16) for $b$ and $q$, we obtain

$$
\begin{gathered}
b=\frac{2\left(1-a^{2}\right)}{a(r-1)}-\frac{(r-1)^{2}}{a}+\frac{3-a^{2}}{a}, \\
q=-\frac{4\left(1-a^{2}\right)}{a^{2}(r-1)^{2}}-\frac{12\left(1-a^{2}\right)}{a^{2}(r-1)} \\
+\frac{3}{a^{2}}\left(4 a^{2}-3\right)+\frac{4}{a^{2}}\left(1+a^{2}\right)(r-1) \\
+\frac{6}{a^{2}}(r-1)^{2}-\frac{1}{a^{2}}(r-1)^{4} .
\end{gathered}
$$

Figure 1 shows $b$ and $q$ as functions of the radius of spherical photon orbits, $r$, in a near-extremal Kerr black hole. Since Eqs. (17) and (18) lead to the inequality $b^{2}>a^{2}-q$, then any spherical photon orbit does not fall in the case of (iii), and therefore $q \geq 0$. It implies that the spherical photon orbits appear in the range

$$
r_{+}<r_{1} \leq r \leq r_{2}, \quad 0<r \leq r_{3}<r_{-},
$$



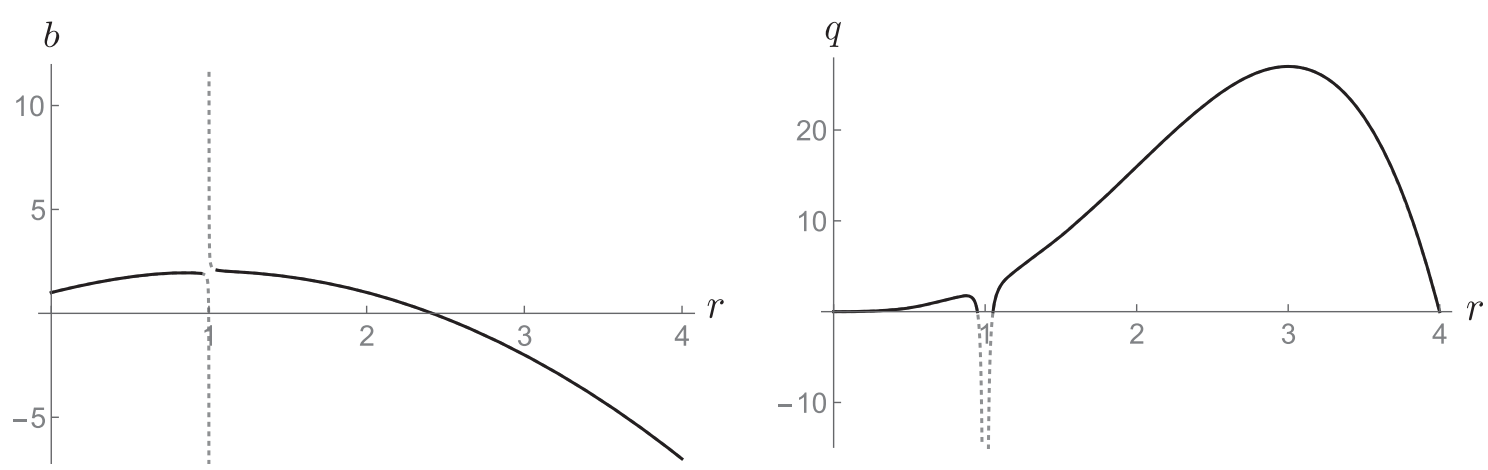

FIG. 1. Plots of $b$ (left) and $q$ (right) as functions of $r$ for $a=0.999$. The solid lines show the values of $b$ and $q$ for each radius of spherical photon orbits in the range $0<r \leq r_{3}$ and $r_{1} \leq r \leq r_{2}$.

where

$$
\begin{gathered}
r_{1}=2+2 \cos \left[\frac{2}{3} \operatorname{Arccos}(a)-\frac{2 \pi}{3}\right], \\
r_{2}=2+2 \cos \left[\frac{2}{3} \operatorname{Arccos}(a)\right], \\
r_{3}=2+2 \cos \left[\frac{2}{3} \operatorname{Arccos}(a)+\frac{2 \pi}{3}\right]
\end{gathered}
$$

are solutions to the equation $q=0$. In the extremal limit, $a \rightarrow 1$, we see that $r_{1} \rightarrow r_{+}$and $r_{3} \rightarrow r_{-}$, and then, on the equatorial plane, there exists a circular photon orbit that approaches the event horizon.

\section{SPHERICAL PHOTON ORBITS THAT APPROACH THE HORIZON IN THE EXTREMAL LIMIT}

For a spherical photon orbit with a radius $r$, a set of impact parameters $b$ and $q$ are given by Eqs. (17) and (18). Then, the polar angle $\theta$ of the orbit varies in the range $\theta_{\min } \leq \theta \leq \theta_{\max }$, where $\theta_{\min / \max }$, are given by

$$
\cos \theta_{\min / \max }=u_{+} \text {. }
$$

With respect to $r$ and $\theta_{\min / \max }$, we plot $(x, z)$ defined by

$x:=\operatorname{sgn}(b) r \sin \theta_{\min / \max }, \quad z:=r \cos \theta_{\min } / \max$

for the spherical photon orbits in a near-extremal black hole in Fig. 2. There are two closed curves: one is outside the outer horizon, and the other is inside the inner horizon. In the extremal limit, $a \rightarrow 1$, the curves in Fig. 2 converge to limit curves consisting of a piece of the circle, $r=1$, which denotes the horizon and the modified cardioid defined by ${ }^{1}$

\footnotetext{
${ }^{1}$ The last term in the right-hand side of Eq. (25) modifies a standard cardioid (see also Ref. [17]).
}

$$
r=1 \pm \sin \theta+\sqrt{2(1 \pm \sin \theta)}
$$

We can classify the spherical photon orbits into two types: the orbits that approach the unit circle, and the orbits that approach the modified cardioid in the $r-\theta_{\min / \max }$ plot as $a \rightarrow 1$. We call the former the "horizon class" and the latter the "cardioid class". A part of the curve outside the horizon makes the cardioid together with a part of the curve inside the horizon. There are the spherical photon orbits of the horizon class both outside the outer horizon and inside the inner horizon. The horizon class and the cardioid class outside the event horizon are joined at the radius $r_{\mathrm{cr}}^{+}:=1+\sqrt[3]{2\left(1-a^{2}\right)}$, while those inside the inner horizon

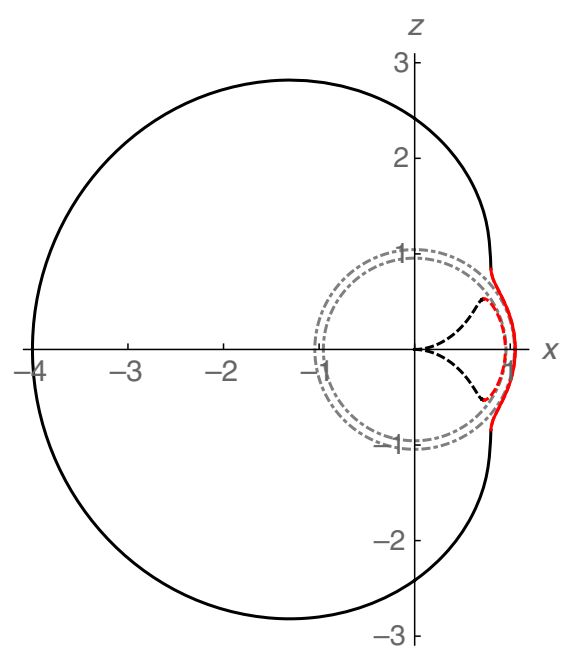

FIG. 2. For the spherical photon orbits in a near-extremal Kerr spacetime with $a=0.999$, the relation of $r$ and $\theta_{\min / \max }$ in the $x-z$ plane is shown, where $(x, z)=\left(\operatorname{sgn}(b) r \sin \theta_{\min / \max }\right.$, $\left.r \cos \theta_{\min / \max }\right)$. The black solid curve corresponds to the cardioid class outside the outer horizon, and the black dashed curve does that inside the inner horizon. The red solid curve and the red dashed curve denote the horizon class outside the outer horizon and inside the inner horizon, respectively. The gray dashed-dotted curves show the outer horizon $r=r_{+}$, and the inner one $r=r_{-}$. 
are joined at the radius $r_{\mathrm{cr}}^{-}:=1-\sqrt[3]{1-a^{2}}$. We should note that the horizon class only appears for $a \geq 1 / \sqrt{2}$.

Here, we concentrate on the spherical photon orbits of the horizon class in the extremal limit, $a \rightarrow 1$. We introduce two small parameters $\epsilon$ and $\delta$ defined by

$$
\epsilon:=1-a, \quad \delta:=r-1,
$$

where $r$ denotes the radius of spherical photon orbits. The radii $r_{1}$ and $r_{3}$ in Eqs. (20) and (22) are expanded by $\epsilon$ as

$$
\begin{aligned}
& r_{1}=1+\sqrt{\frac{8}{3}} \epsilon^{1 / 2}+O(\epsilon) \quad \text { and } \\
& r_{3}=1-\sqrt{\frac{8}{3}} \epsilon^{1 / 2}+O(\epsilon),
\end{aligned}
$$

respectively. These, together with Eq. (19), imply that

$$
\epsilon \ll \sqrt{\frac{8}{3}} \epsilon^{1 / 2} \leq|\delta| \ll 1 .
$$

Then, we can expand $b$ and $q$ in terms of $\epsilon$ and $\delta$ as

$$
b \simeq 2+\frac{4 \epsilon}{\delta}+4 \epsilon-\delta^{2}, \quad q \simeq 3-\frac{8 \epsilon}{\delta^{2}}+8 \delta-\frac{24 \epsilon}{\delta} .
$$

If we take the limits $\epsilon \rightarrow 0$ and $|\delta| \rightarrow 0$ under the condition

$$
\epsilon \ll \sqrt{\frac{8}{3}} \epsilon^{1 / 2}=|\delta| \ll 1,
$$

then we have

$$
b \rightarrow 2, \quad q \rightarrow 0
$$

On the other hand, if we take the limits $\epsilon \rightarrow 0$ and $|\delta| \rightarrow 0$ under the condition

$$
\epsilon \ll \epsilon^{1 / 2} \ll|\delta| \ll 1,
$$

then we have

$$
b \rightarrow 2, \quad q \rightarrow 3 .
$$

Hence, for the spherical photon orbits that approach the horizon, $\delta \rightarrow 0$, in the extremal limit, $\epsilon \rightarrow 0$, the parameter $b$ approaches 2 , and the parameter $q$ takes a value in the range $0 \leq q \leq 3$.

In the extremal limit, $a \rightarrow 1, \theta_{\min / \max }$ of the spherical photon orbits of the horizon class is given by

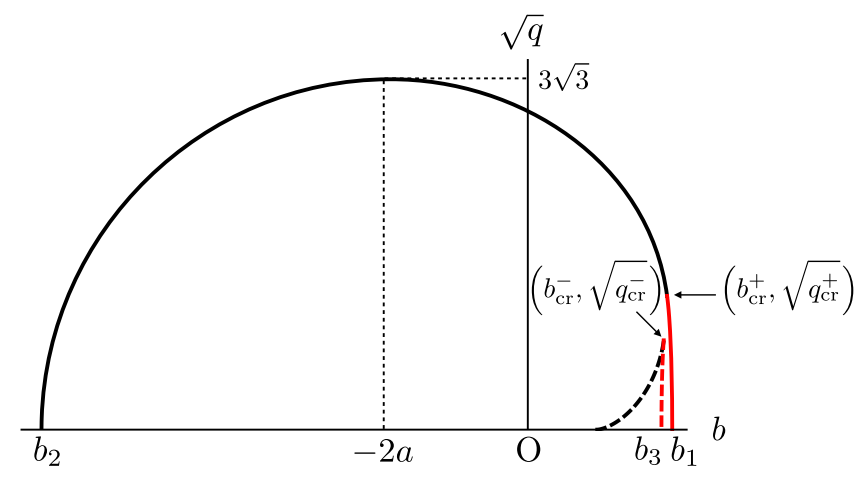

FIG. 3. The relation of $b$ and $\sqrt{q}$ is shown for spherical photon orbits in the case of $a=0.999$. The solid curve corresponds to the spherical photon orbits in the range $r_{+}<r_{1} \leq r \leq r_{2}$, and the dashed curve does to the ones in $0<r \leq r_{3}<r_{-}$. The photon orbits with $(b, \sqrt{q})=\left(b_{1}, 0\right),\left(b_{2}, 0\right)$ are the prograde circular orbit at $r=r_{1}$ and the retrograde circular orbit at $r=r_{2}$ on the equatorial plane, respectively. The photon orbit with $\left(b_{3}, 0\right)$ is the prograde circular orbit inside the inner horizon at $r=r_{3}$. In the limit $a \rightarrow 1$, the black solid curve and the black broken curve converge to a cardioid, and the red solid curve and the red broken curve do to a straight segment.

$$
\begin{aligned}
\left|\cos \theta_{\min / \max }\right| & =\frac{1}{\sqrt{2}}[\sqrt{(q+1)(q+9)}-q-3]^{1 / 2} \\
& \leq \sqrt{2 \sqrt{3}-3}
\end{aligned}
$$

where the inequality is evaluated by $q=3$. This value was also found by the analysis of the near horizon of the extremal Kerr geometry in Refs. [18-21] and Ref. [22] in a different context.

We can find another cardioid for the spherical photon orbits in the relation between the impact parameters given by Eqs. (17) and (18) as curves in the $b-\sqrt{q}$ plane as shown in Fig. 3. In the extremal limit, $a \rightarrow 1$, the curves converge to the cardioid that is expressed by using a parameter $\psi$ as

$$
\begin{aligned}
b-1 & =4(1-\cos \psi) \cos \psi \\
\sqrt{q} & =4(1-\cos \psi) \sin \psi
\end{aligned}
$$

and the straight segment that connects $(b, \sqrt{q})=(2, \sqrt{3})$ and $(2,0)$. The horizon class corresponds to the straight segment, while the cardioid class does to the cardioid in the $b-\sqrt{q}$ plane. On the curves in Fig. 3, the horizon class and the cardioid class are joined at critical points $b_{\mathrm{cr}}^{ \pm}, q_{\mathrm{cr}}^{ \pm}$ defined by $b_{\mathrm{cr}}^{+}:=3 / a-a, \quad q_{\mathrm{cr}}^{+}:=6\left[2+\sqrt[3]{2\left(1-a^{2}\right)}\right]-$ $9 / a^{2}, \quad$ and $\quad b_{\mathrm{cr}}^{-}:=(3 / a)\left[1-\sqrt[3]{\left(1-a^{2}\right)^{2}}\right]-a, \quad q_{\mathrm{cr}}^{+}:=$ $\left(3 / a^{2}\right)\left[1-\sqrt[3]{1-a^{2}}\right]^{4}$, respectively. 


\section{WEYL CURVATURE NEAR THE HORIZON OF A NEAR-EXTREMAL Kerr SPACETIME}

We consider a property of the Weyl curvature near the horizon of the near-extremal Kerr geometry. To introduce a parallelly propagated tetrad along a null geodesic [23], we use the conformal Killing-Yano 2-form

$$
\begin{aligned}
h= & r\left(\mathrm{~d} t-a \sin ^{2} \theta \mathrm{d} \varphi\right) \wedge \mathrm{d} r \\
& +a \cos \theta \sin \theta\left[a \mathrm{~d} t-\left(r^{2}+a^{2}\right) \mathrm{d} \varphi\right] \wedge \mathrm{d} \theta
\end{aligned}
$$

and the Killing-Yano 2-form

$$
\begin{aligned}
f= & a \cos \theta\left(\mathrm{d} t-a \sin ^{2} \theta \mathrm{d} \varphi\right) \wedge \mathrm{d} r \\
& -r \sin \theta\left[a \mathrm{~d} t-\left(r^{2}+a^{2}\right) \mathrm{d} \varphi\right] \wedge \mathrm{d} \theta .
\end{aligned}
$$

These forms yield parallelly propagated unit spacelike vectors orthonormal to $k^{a}$,

$$
m^{a}=\frac{k^{b} h_{b}{ }^{a}-\lambda\left(\xi_{c} k^{c}\right) k^{a}}{\sqrt{C_{c d} k^{c} k^{d}}}, \quad n^{a}=\frac{k^{b} f_{b}{ }^{a}}{\sqrt{K_{c d} k^{c} k^{d}}},
$$

and a null vector external to $k^{a}$,

$l^{a}=\frac{m^{b} h_{b}{ }^{a}}{\sqrt{C_{c d} k^{c} k^{d}}}+\frac{C_{b}{ }^{d} C_{d c} k^{b} k^{c}+\lambda^{2}\left(\xi_{e} k^{e}\right)^{2} C_{c d} k^{c} k^{d}}{2\left(C_{c d} k^{c} k^{d}\right)^{2}} k^{a}$,

where $\xi^{a}=(1 / 3) \nabla_{b} h^{b a}=(\partial / \partial t)^{a}$ is the stationary Killing vector, $K_{a b}=f_{a c} f_{b}{ }^{c}$ coincides with the Killing tensor in Eq. (5), and $C_{a b}=h_{a c} h_{b}{ }^{c}$ is a conformal Killing tensor.

The parallelly propagated tetrad $\left\{k^{a}, l^{a}, m^{a}, n^{a}\right\}$ along a null geodesic becomes singular due to an infinite gravitational blue shift if the geodesic approaches the horizon. Simultaneously, the divergence of $k^{\phi}$ means that a photon orbits the black hole infinite times during a finite interval of the affine parameter. In order to estimate the shear of a congruence of null geodesics while they wind finite times around the black hole, we should regularize the tetrad even on the horizon. Then, we introduce

$$
\tilde{k}^{a}=\sqrt{\Delta} k^{a}, \quad \tilde{l}^{a}=\frac{l^{a}}{\sqrt{\Delta}} .
$$

Note that $\tilde{k}^{a}$ and $\tilde{l}^{a}$ are parallelly propagated if we restrict the null geodesics to spherical photon orbits.

We evaluate the tetrad components of the Weyl tensor $C_{\tilde{k} A B \tilde{k}}:=C_{a b c d} \tilde{k}^{a}\left(e_{A}\right)^{b}\left(e_{B}\right)^{c} \tilde{k}^{d}$ on the spherical photon orbits, where $\left(e_{1}\right)^{a}=m^{a}$ and $\left(e_{2}\right)^{a}=n^{a}$. Using Eqs. (17) and (18), we have

$$
C_{\tilde{k} 11 \tilde{k}}=-C_{\tilde{k} 22 \tilde{k}}=\frac{12 r^{3} \Delta^{2}\left[5\left(r^{2}-a^{2} \cos ^{2} \theta\right)^{2}-4 r^{4}\right]}{(r-1)^{2} \Sigma^{5}},
$$

$$
\begin{aligned}
C_{\tilde{k} 12 \tilde{k}} & =C_{\tilde{k} 21 \tilde{k}} \\
& =\frac{12 a r^{2} \Delta^{2} \cos \theta\left[5\left(r^{2}-a^{2} \cos ^{2} \theta\right)^{2}-4 a^{4} \cos ^{4} \theta\right]}{(r-1)^{2} \Sigma^{5}} .
\end{aligned}
$$

It is clear that these Weyl components are nonvanishing for the spherical photon orbits of the cardioid class, where $r>1$. In contrast, for the spherical photon orbits of horizon class, taking the limit $\epsilon \rightarrow 0$ and $\delta \rightarrow 0$, we find

$$
\begin{aligned}
C_{\tilde{k} 11 \tilde{k}} & =-C_{\tilde{k} 22 \tilde{k}} \\
& \simeq \frac{12\left(1-10 \cos ^{2} \theta+5 \cos ^{4} \theta\right)}{\left(1+\cos ^{2} \theta\right)^{5}}\left(\delta^{2}-4 \epsilon\right) \rightarrow 0, \\
C_{\tilde{k} 12 \tilde{k}} & =C_{\tilde{k} 21 \tilde{k}} \\
& \simeq \frac{12 \cos \theta\left(5-10 \cos ^{2} \theta+\cos ^{4} \theta\right)}{\left(1+\cos ^{2} \theta\right)^{5}}\left(\delta^{2}-4 \epsilon\right) \rightarrow 0 .
\end{aligned}
$$

With the assumption that the congruence is twist-free, the evolution of the expansion, $\Theta$, and the shear, $\sigma_{A B}$, of a congruence of spherical photon orbits in Kerr spacetimes are determined by

$$
\begin{aligned}
\frac{\mathrm{d}}{\mathrm{d} \tilde{\lambda}} \Theta & =-\frac{\Theta^{2}}{2}-\sigma^{A B} \sigma_{A B}, \\
\frac{\mathrm{d}}{\mathrm{d} \tilde{\lambda}} \sigma_{A B} & =-\Theta \sigma_{A B}+C_{\tilde{k} A B \tilde{k}},
\end{aligned}
$$

where $\tilde{\lambda}$ is a affine parameter on the spherical photon orbit. Then, Eqs. (43) and (44) mean that the Weyl curvature does not produce the shear of a congruence of spherical photon orbits of the horizon class in near-extremal Kerr black holes. Hence, if the congruence has the initial conditions $\Theta=0$ and $\sigma_{A B}=0$, then $\Theta$ and $\sigma_{A B}$ remains zero along the orbit.

In the extremal limit, $a \rightarrow 1$, we see, from Eqs. (8) and (40), that $\tilde{k}^{a}$ of a spherical photon orbit of the horizon class approaches the horizon generator $\chi^{a}$, given by Eq. (3). On the other hand, the regularized outgoing principal null vector $(\Delta / 2) N_{+}^{a}$ on the horizon is proportional to $\chi^{a}$. Although $(\Delta / 2) N_{+}^{a}$ and $\tilde{k}^{a}$ are characterized by different values of the constants of motion $(b, q)$, both vectors approach the unique null vector on the event horizon $\chi^{a}$ in the limit $a \rightarrow 1$ and $r \rightarrow 1$. Therefore, we can understand that the spherical photon orbit of the horizon class is shearfree in this limit from the fact that the principal null geodesic is shear-free in the Kerr spacetime, which is classified in Petrov type D. 


\section{SUMMARY AND DISCUSSIONS}

Vanishing shear of a congruence of the spherical photon orbits of the horizon class is interesting from the observational point of view. An image of a compact source through a congruence of light rays in the horizon class around a near-extremal Kerr black hole can keep its brightness even if the photons orbit around the black hole many times, and then, it would be observable. For extended sources, the spherical photon orbits correspond to the bright border of the black hole shadow [3-8,18,24-27]. In this case, photon orbits of the horizon class and the ones of the cardioid class would make high contrast of brightness of the shadow border for an equatorial observer. It is an interesting and important next work to clarify the relation quantitatively between the contrast and spin parameter of the black hole.

\section{ACKNOWLEDGMENTS}

The authors thank T. Harada, S. Kinoshita, T. Koike, K. Nakao, J. M. M. Senovilla, R. Takahashi, and Y. Yasui for useful comments. This work was supported by Grant-in-Aid for Early-Career Scientists [JSPS KAKENHI Grant No. JP19K14715] (T. I.) and Grant-inAid for Scientific Research (C) [JSPS KAKENHI Grant No. JP16K05358] (H. I.) from the Japan Society for the Promotion of Science and the MEXT-Supported Program for the Strategic Research Foundation at Private Universities, 2014-2017 (S1411024) from the Ministry of Education, Culture, Sports, Science and Technology (T. I.).
[1] J. Wambsganss, Living Rev. Relativity 1, 12 (1998).

[2] E. Teo, Gen. Relativ. Gravit. 35, 1909 (2003).

[3] K. Akiyama et al. (Event Horizon Telescope Collaboration), Astrophys. J. 875, L1 (2019).

[4] K. Akiyama et al. (Event Horizon Telescope Collaboration), Astrophys. J. 875, L2 (2019).

[5] K. Akiyama et al. (Event Horizon Telescope Collaboration), Astrophys. J. 875, L3 (2019).

[6] K. Akiyama et al. (Event Horizon Telescope Collaboration), Astrophys. J. 875, L4 (2019).

[7] K. Akiyama et al. (Event Horizon Telescope Collaboration), Astrophys. J. 875, L5 (2019).

[8] K. Akiyama et al. (Event Horizon Telescope Collaboration), Astrophys. J. 875, L6 (2019).

[9] C. S. Reynolds, Space Sci. Rev. 183, 277 (2014).

[10] J. M. Bardeen, W. H. Press, and S. A. Teukolsky, Astrophys. J. 178, 347 (1972).

[11] J. M. Bardeen and G. T. Horowitz, Phys. Rev. D 60, 104030 (1999).

[12] M. Guica, T. Hartman, W. Song, and A. Strominger, Phys. Rev. D 80, 124008 (2009).

[13] G. Compére, Living Rev. Relativity 15, 11 (2012); 20, 1 (2017).
[14] S. E. Gralla, A. Lupsasca, and A. Strominger, Mon. Not. R. Astron. Soc. 475, 3829 (2018).

[15] A. Lupsasca, A. P. Porfyriadis, and Y. Shi, Phys. Rev. D 97, 064017 (2018).

[16] B. Carter, Phys. Rev. 174, 1559 (1968).

[17] O. Y. Tsupko, Phys. Rev. D 95, 104058 (2017).

[18] J. M. Bardeen, Timelike and null geodesics in the Kerr metric, in Black Holes (Les Astres Occlus), edited by C. Dewitt and B.S. Dewitt (Gordon and Breach, NewYork, 1973), pp. 215-239.

[19] A. M. Al Zahrani, V. P. Frolov, and A. A. Shoom, Int. J. Mod. Phys. D 20, 649 (2011).

[20] S. Hod, Phys. Lett. B 718, 1552 (2013).

[21] A. P. Porfyriadis, Y. Shi, and A. Strominger, Phys. Rev. D 95, 064009 (2017).

[22] T. Harada and M. Kimura, Phys. Rev. D 83, 084041 (2011).

[23] D. Kubiznak, V. P. Frolov, P. Krtous, and P. Connell, Phys. Rev. D 79, 024018 (2009).

[24] P. J. Young, Phys. Rev. D 14, 3281 (1976).

[25] H. Falcke, F. Melia, and E. Agol, Astrophys. J. 528, L13 (2000).

[26] S. Doeleman et al., Nature (London) 455, 78 (2008).

[27] S. S. Doeleman et al., Science 338, 355 (2012). 